\title{
An Existence Theorem for Positive Solutions of Semilinear Elliptic Equations
}

\author{
Joel SMOller \& Arthur Wasserman
}

Communicated by J. SERRIN

1.

In this paper we consider the existence of positive solutions of the Dirichlet problem

$$
\begin{aligned}
\Delta u(x)+f(u(x)) & =0, \quad x \in D^{n} \\
u(x) & =0, \quad x \in \partial D^{n},
\end{aligned}
$$

where $D^{n}$ is an $n$-ball of radius $R$ in $\mathbb{R}^{n}$. The well-known identity of PoHozaEV [4] shows that the existence question, even for simple functions such as $f(u)=u^{k}$, is quite delicate. Previous results have been obtained by placing severe restrictions on $f$; for example, in [3] it is required that $f(0) \geqq 0$, and in [1] one finds $f^{\prime}(0)>0$, and $f(u) \geqq 0$.

Our approach is based upon the celebrated GidAs, NI, \& NIRENBERG Theorem, [2], which asserts that any positive solution of (1) must be radially symmetric, and thus satisfies an ordinary differential equation. In this context, it is natural to allow the radius $R$ of $D^{n}$ to vary, and to consider orbits of the associated ordinary differential equation which satisfy $u=p>0$ and $u^{\prime}=0$ at $r=0$. Then we may define $T(p)$ to be the smallest $R>0$ for which this orbit satisfies $u(R)=0$.

In this note, we show that existence results may be obtained for very general functions $f$. Specifically, if $f\left(p_{0}\right)=0$ for some $p_{0}>0$, we show that there exists a positive solution of (1), with $u(0)$ close to $p_{0}$ and $u(0)<p_{0}$, for some $R$, if and only if there exists a positive solution of the Dirichlet problem

$$
u^{\prime \prime}(x)+f(u(x))=0, \quad x \in D^{\mathbf{1}}
$$

$$
u(x)=0, \quad x \in \partial D^{1},
$$

with $u(0)$ close to $p_{0}$ and $u(0)<p_{0}$, for some $R$. Since this latter problem is equivalent to solving a Hamiltonian system having one-degree of freedom, it 
can be solved by phase-plane methods. In fact, it is easy to show that there exists a solution of (2), with $u(0)$ near $p_{0}$ and $u(0)<p_{0}$, for some $R$, if and only if

$$
\int_{p}^{p_{0}} f(u) d u>0
$$

for $0 \leqq p<p_{0}$. If we define

$$
F(u)=\int_{0}^{u} f(s) d s,
$$

then we can state our theorem as follows.

Theorem. Let $f \in C^{1}$ and suppose that $f\left(p_{0}\right)=0$ for some $p_{0}>0$. Then the following statements are equivalent.

(A) There exist positive solutions of (1) for which

$$
u(0) \text { is arbitrarily close to } p_{0} \text {; }
$$

(B) There exist positive solutions of (2) for which (4) holds;

(C) $F(p)<F\left(p_{0}\right)$ for $0 \leqq p<p_{0}$.

Observe that we place no conditions on the sign of $f(0)$, nor do we require that $0<f(u)$ if $0<u<\beta$, for some $\beta>0$; cf. [3].

In the next section we shall give the proof of the theorem, and in section 3 we shall derive some consequences of our result.

\section{2.}

From a result of GIDAS, NI, \& NiRENBERG [2] the existence of a positive solution of (1) on a ball is equivalent to the existence of a positive solution for a boundary-value problem of the form

$$
\begin{gathered}
u^{\prime \prime}(r)+\frac{n-1}{r} u^{\prime}(r)+f(u(r))=0, \quad 0<r<T \\
u^{\prime}(0)=u(T)=0 .
\end{gathered}
$$

We let $u(r, p)$ be the solution of (5) which satisfies the initial conditions $u(0)=p$, $u^{\prime}(0)=0$.

We first show that (A) implies (C). As we have already noted, it suffices to assume that (5), (6) has solutions (for some $T=T(p)$ ) which satisfy (4). Now if (C) were false, let $q<p_{0}$ be a point where the maximum of $F$ on the interval $\left[0, p_{0}\right]$ is assumed. Then $F(q) \geq F(p)$ for $p$ near $p_{0}$ with $p<p_{0}$. Using (A), we know that, for some such $p$, there is a solution $u(r, p)$ of (5) satisfying $u^{\prime}(0, p)=0, u(0, p)=p$ and $u(T(p), p)=0$. It follows that there is an $r_{1}>0$ such that $u\left(r_{1}, p\right)=q$. Then if $H(u, v)=F(u)+v^{2} / 2,\left(v=u^{\prime}\right)$, we see that $H^{\prime}=(1-n) u^{\prime}(r, p)^{2} / r \leqq 0$ along this solution. But

$$
H\left(u\left(r_{1}, p\right), u^{\prime}\left(r_{1}, p\right)\right)>F(q) \geq F(p)=H\left(u(0, p), u^{\prime}(0, p)\right),
$$


and this is a contradiction. Thus (A) implies (C). In a similar manner, we can show that (B) implies (C).

We next prove that $(C)$ implies (B). Thus, define

$$
M=\{p: F(q)<F(p) \quad \text { for all } q, 0 \leqq q<p\} \cap\left(0, p_{0}\right]
$$

then $M \neq \emptyset$ since $p_{0} \in M$. We claim that there are points $p \in M$ arbitrarily close to $p_{0}$, with $f(p)>0$. To see this, let $\delta>0$ be given; we must show that there is a point $p \in M \cap\left[p_{0}-\delta, p_{0}\right]$, with $f(p)>0$. Let

$$
a=\sup \left\{F(q): 0 \leqq q \leqq p_{0}-\delta\right\} ;
$$

then $a<F\left(p_{0}\right)$. Choose $x$ so that $a<x<F\left(p_{0}\right)$ and set

$$
y=\inf \left\{q: F(q)=x, \quad \text { and } \quad p_{0}-\delta<q<p_{0}\right\} .
$$

Then clearly both $F(y)=x$ and $y \in\left[p_{0}-\delta, p_{0}\right]$. But also $y \in M$, since if $0 \leqq q<y$ and $0 \leqq q \leqq p_{0}-\delta$, then $a \geqq F(q)$. Consequently $a<x$ implies $F(y)=x>F(q)$. On the other hand, if $p_{0}-\delta<q<p_{0}$, then $q<y$ implies $F(q) \neq x$. If $F(q)>x$, then since $x>a$ we could find $\bar{q}<q$ with $F(\bar{q})=x$ and this violates the definition of $y$; thus again $x>F(q)$. Hence $y \in M \cap$ $\left[p_{0}-\delta, p_{0}\right)$. Thus $F\left(M \cap\left[p_{0}-\delta, p_{0}\right] \supset\left(a, F\left(p_{0}\right)\right]\right.$ implies that meas $\{F(M \cap$ $\left.\left[p_{0}-\delta, p_{0}\right)\right\}>0$, and the claim follows from SARD's Theorem.

Take $p \in M \cap\left[p_{0}-\delta, p_{0}\right)$ with $f(p)>0$. Then the orbit of (2) through $(p, 0)$ enters the region $\{u>0, v<0\}$. Since $H$ is constant along this orbit, we have $F(p)=F(u)+v^{2} / 2$ for $u<p$. Since $p \in M$, this orbit cannot leave the region $\{u \geqq 0, v \leqq 0\}$ through the line $v=0$, and so it must leave through a point $(0, v)$ with $v<0$. Thus there is a solution of (2) with $u(0)=p \in\left[p_{0}-\delta\right.$, $\left.p_{0}\right)$. Therefore since $\delta$ was arbitrary, we see that (B) holds.

In order to complete the proof of the theorem, we shall prove that $(C)$ imp lie (A). Let $\delta>0$ be given and choose $q \in M \cap\left[p_{0}-\delta, p_{0}\right)$ with $f(q)>0$, ass above. Let

$$
\bar{q}=\min \left\{u: q<u \leqq p_{0}, \quad \text { and } \quad f(u)=0\right\} .
$$

We shall find a solution of (5), (6) with $q<u(0)<\bar{q}$. We define positive constants $\varepsilon, \bar{M}, t_{0}$, as follows:

$$
\begin{gathered}
3 \varepsilon=F(\bar{q})-F(q)=\int_{q}^{\bar{q}} f(u) d u \\
\vec{M}=\sqrt{2}\left[F(\bar{q})-\min _{0 \leqq s \leqq \bar{q}} F(s)\right]^{1 / 2} \\
t_{0}=(n-1) M \bar{q} / \varepsilon .
\end{gathered}
$$

Set

$$
H(r, p)=F(u(r, p))+u^{\prime}(r, p)^{2} / 2
$$


then $H_{r}=(1-n) u^{\prime}(r, p)^{2} / r \leqq 0$. We choose $p$, with $q<p<\bar{q}$, such that both

$$
u\left(t_{0}, \bar{q}\right)-u\left(t_{0}, p\right)<\bar{q}-q \quad \text { and } \quad H\left(t_{0}, \bar{q}\right)-H\left(t_{0}, p\right)<\varepsilon ;
$$

this can be done since $u\left(t_{0}, \bar{q}\right)=\bar{q}$ and since $H$ is continuous.

We now show that $u(T, p)=0$ for some $T>0$. Because $f(p)>0$, we see, as above, that the orbit through $u=p, v=0$ enters the region $\{u>0$, $v<0\}$. Also, since $f$ is bounded away from zero on $[q, p]$, there is a constant $t_{1}>0$ such that $u\left(t_{1}, p\right)=q$ and $u(r, p) \geqq q$ and $v(r, p) \equiv u^{\prime}(r, p) \leqq 0$ for $0 \leqq r \leqq t_{1}$; see [6, Theorem 17]. Note also that $t_{1}>t_{0}$ since $u\left(t_{0}, p\right)>q$.

We shall show that $u(T, p)=0$ for some $T$ satisfying $t_{1}<T \leqq t_{2}=$ $t_{1}+q / \sqrt{2 \varepsilon}$. To see this, observe that, for $r>0$,

$$
F(p)=H(0, p) \geqq H(r, p)=F(u(r, p))+v^{2}(r, p) / 2 .
$$

Hence $v^{2} \leqq 2(F(p)-F(u)) \leqq \bar{M}^{2}$, so $|v| \leqq \bar{M}$. We also claim that if $t_{2} \geqq r \geqq t_{1}$ then $-v(r, p) \geqq \sqrt{2 \varepsilon}$. Assuming this, we have

$$
u\left(t_{2}, p\right)=u\left(t_{1}, p\right)+\int_{t_{1}}^{t_{2}} v(r, p) d r \leqq q+\int_{i_{1}}^{t_{2}}-\sqrt{2 \varepsilon} d r=0,
$$

which implies the desired conclusion. Therefore to complete the proof it suffices to prove the above claim. Thus, assume that for some $t, t_{1} \leqq t \leqq t_{2}$, we have $v(t, p)>-\sqrt{2 \varepsilon}$ and $v(r, p) \leqq 0$ for $0 \leqq r \leqq t$. Then

$$
\begin{aligned}
\frac{v(t, p)^{2}}{2} & =H(t, p)-F(u(t, p)) \\
& =H(t, p)-H\left(t_{0}, p\right)+H\left(t_{0}, p\right)-F(u(t, p)) \\
& =\int_{t_{0}}^{t} H_{r}(r, p) d r+H\left(t_{0}, p\right)-F(u(t, p)) \\
& =-\int_{t_{0}}^{t} \frac{n-1}{r} v^{2}(r, p) d r+H\left(t_{0}, p\right)-F(u(t, p)) \\
& \geqq \frac{(n-1) \bar{M}}{t_{0}} \int_{t_{0}}^{t} v(r, p) d r+H\left(t_{0}, \bar{q}\right)-\varepsilon-F(u(t, p)) \\
& \geqq \frac{(n-1) \bar{M}}{t_{0}}\left[u(t, p)-u\left(t_{0}, p\right)\right]+F(\bar{q})-\varepsilon-F(u(t, p)) \\
& \geqq \frac{(n-1) \bar{M}}{t_{0}}(-q)+F(q)-F(u(t, p))+2 \varepsilon \\
& \geqq \varepsilon+F(q)-F(u(t, p)) .
\end{aligned}
$$

But because $t \geqq t_{1}$ we have $u(t, p) \leqq q$. Hence $q \in M$ implies that $F(q)>$ $F(u(t, p))$. It follows that $v^{2}(t, p) \geqq 2 \varepsilon$. Consequently, since $v(t, p) \leqq 0$, we 
have $-v(t, p) \geqq \sqrt{2 \varepsilon}$. This is a contradiction, and the claim follows. The proof of the theorem is complete.

Notice that if $f^{\prime}\left(p_{0}\right)<0$, then we can strengthen (4) to the following:

$$
u(0) \text { is in }\left[p_{0}-\delta, p_{0}\right) \text { for some } \delta>0 \text {. }
$$

To see this, observe that if $f^{\prime}\left(p_{0}\right)<0$ then there is a number $\delta_{1}>0$ such that $f(p)>0, p_{0}-\delta_{1}<p<p_{0}$. Thus we can let $\bar{q}=p_{0}-\delta_{1}$.

3.

In this section we shall add some remarks and state several consequences of our theorem.

First, we observe that in some cases our theorem can be used to deduce the existence of other solutions. For example, assume that $f(0) \geqq 0$, and suppose that there are points $p_{0}$ and $p_{1}, p_{0}<p_{1}$, both of which satisfy hypothesis (C) of the theorem. We claim that for balls of sufficiently large radii, there must be at least two positive solutions. To see this, take $\tilde{p}, p_{0}<\tilde{p}<p_{1}$, with the property that there is a positive solution of (5), (6) with $u(0)=\tilde{p}$. Then $v(T, \tilde{p})<0$ since $f(0) \geqq 0$. Thus $u(T, \tilde{p})=0$ and $u^{\prime}(T, \tilde{p})=v(T, \tilde{p}) \neq 0$, so by the implicit function theorem $T$ is a function of $p$ for $p$ near $\tilde{p}$. Hence there is a neighborhood about $\tilde{p}$ which is contained in the domain of $T$. Let $(\alpha, \beta)$ be the maximal interval about $\tilde{p}$ which is in the domain of $T$. Then $p_{0} \leqq \alpha<\tilde{p}<\beta \leqq p_{1}$ and

$$
\varlimsup_{p \backslash x} T(p)=\varlimsup_{p, \pi \beta} T(p)=\infty \text {. }
$$

(If for example $\varlimsup_{p \nearrow \beta} T(p)=M<\infty$, then we could find points $p_{n} \nearrow \beta$ such that $T\left(p_{n}\right) \rightarrow T_{0}<\infty$. But $u\left(T\left(p_{n}\right), p_{n}\right)=0$ implies $u\left(T_{0}, \beta\right)=0$, and this violates the definition of $\beta$ since $\left.u^{\prime}\left(T_{0}, \beta\right) \neq 0\right)$. It follows that, for $T>T(\tilde{p})$, there exists at least two solutions. Thus we have the following

Corollary. Let $f \in C^{1}$ and $f(0) \geqq 0$, and suppose there are points $p_{0}<p_{1}$ such that

$$
f\left(p_{i}\right)=0, \quad F(p)<F\left(p_{1}\right) \quad \text { for } \quad 0 \leqq p<p_{1} .
$$

Then there is an $\bar{R}$ such that if $R>R$ the problem (1) has at least two positive solutions on the ball of radius $R$. These solutions satisfy $p_{0}<u(0)<p_{1}$.

As another application of our theorem, we shall show that the conclusion of the last corollary (i.e., the existence of a second solution) can hold under quite different hypotheses. For example, suppose that $k \geq \frac{n+2}{n-2}$ and that $f$ is a smooth function such that $f(u)=u^{k}, 0 \leqq u \leqq a, f(b)=0, f^{\prime}(b)<0,0<a<b$, and $f(u)>0$. for $0<u<b$.

Then our theorem shows that (5), (6) has solutions for $p$ near $b$. If we define $\bar{p}=\inf \{p: 0<p<b$ and (5), (6) is solvable for some $T>0$, with $u(o)=p\}$, 
then $a<\bar{p}<b$ in view of the well-known PoHOzAEv identity; see [3]. Taking any $p$ in the domain of $T$ which satisfies $\bar{p}<p<b$, we see that the last argument holds. It follows that for large $R$ the Dirichlet problem (1) again has at least two positive solutions.

We end by noting that if $f(u)>0$ for $u<0$ then all solutions of (1) are positive and hence radial. To see this, observe that if there is an $x \in \Omega$ with $u(x)<0$, then

$$
\inf \{u(x): x \in \Omega\}
$$

is attained at a point $\bar{x}$ in $\Omega$. At this point, $\nabla u(\bar{x})=0$ and $\Delta u(\bar{x}) \geqq 0$ and we obtain the contradiction $\Delta u(\bar{x})+f(u(\bar{x}))>0$. This observation, together with our previous remarks, can be applied, for example, to the cubic nonlinear function

$$
f(u)=-u(u-a)(u-1), \quad 0<a<1 / 2 .
$$

Hence, for this $f$, all solutions of (1) are radial, and (1) has at least two solutions if the radius of $D^{n}$ is sufficiently large. It follows that problem (1) has at least one degenerate solution, in the sense that 0 is in the spectrum of the linearized equation; see [6]. It would be interesting to determine the number of critical points of the time map $T(p)$ for $f$ defined by (8). In [5] we have shown that if $n=1$ then $T$ has exactly one critical point.

Note added in proof. After this paper was accepted, we learned of the paper of P. Hess (On multiple solutions of nonlinear elliptic eigenvalue problems, Comm. on Partial Differential Equations 6, 1981, 951-961) in which similar results are obtained. Hess requires stronger conditions on $f$ (including $f(0)>0$ ) but he allows more general domains.

Acknowledgement. This research was partially supported by National Science Foundation under Grant No. MCS-8002337.

\section{References}

1. AmanN, H., Fixed point equations and nonlinear eigenvalue problems in ordered Banach spaces, SIAM Rev., 18, (1976), 620-709.

2. Gidas, B., W. N. Ni, \& L. NirenBerG, Symmetry of positive solutions of nonlinear elliptic equations in $\mathbb{R}^{n}$, Comm. Math. Phys., 68, (1979), 209-243.

3. LIONS, P. L., On the existence of positive solutions of semilinear elliptic equations, SIAM Rev., 24, (1982), 441-467.

4. Pohozaev, S. I., Eigenfunctions of the equation $\Delta u+\lambda f(u)=0$, Sov. Math. Dok., 5, (1965), 1408-1411.

5. Smoller, J., \& A. Wasserman, Global bifurcation of steady-state solutions, J. Diff. Equations, 39, (1981), 269-290.

6. Smoller, J., \& A. WASSERman, Existence, uniqueness, and nondegeneracy of positive solutions of semilinear elliptic equations, Comm. Math. Phys., 95, (1984), 129-159.

\section{Department of Mathematics University of Michigan Ann Arbor}

